\title{
Genetic Algorithm for Line Labeling of Diagrams Having Drawing Cues
}

Conference Paper · July 2012

DOI: 10.1007/978-3-642-31223-6_41

CITATIONS

2

2 authors:

\section{Alexandra Bonnici}

University of Malta

27 PUBLICATIONS 72 CITATIONS

SEE PROFILE
READS

30

Some of the authors of this publication are also working on these related projects: 


\title{
Genetic Algorithm for Line Labeling of Diagrams having Drawing Cues
}

\author{
Alexandra Bonnici and Kenneth Camilleri \\ Department of Systems and Control Engineering, University of Malta, Malta \\ alexandra.bonnici@um.edu.mt, kenneth.camilleri@um.edu.mt
}

\begin{abstract}
Drawings are an integral part of the design process, helping designers communicate abstract concepts to others. In this paper we propose a genetic algorithm that successfully exploits cues present in drawings in a line labeling algorithm for sketches.
\end{abstract}

Keywords: genetic algorithms, line labeling, drawing cues

\section{Introduction}

Cues help artists portray intent and hence aid the drawing interpretation. Cues may include line phrasing where designers adjust the stroke width according to the depth of the object edge[3], table lines which indicate the spatial relation of the object with respect to its background and tone or illumination changes. In pen-and-paper sketches, tone changes are created by hatching techniques [4] and serve to give the impression of depth as well as to emphasize the shape and form of the object and its spatial relationship with other objects in the sketch. Here we propose a method to exploit such cues in an off-line line labeling algorithm suitable for hand-drawn sketches.

\section{Genetic Algorithm Approach for Line Labeling}

Line labeling algorithms are used to describe each edge in the drawing in relation to its neighbouring edges. Huffman $[5]$ and Clowes [1] created a junction dictionary $\boldsymbol{\Gamma}$ by which these edge can be labeled. Waltz [8] and Cooper [2] enhanced the labeling by introducing hard constraints that determine the appropriate edge label in scenes containing shadows and contrast failures. However, hard constraints are inappropriate for use with concept sketches where cues may be geometrically incorrect. Hancock and Myers [7] propose the use of a genetic algorithm (GA) to determine the edge labels, representing edges as an arbitrarily ordered sequence of genes forming a fixed length chromosome. Each gene may take a value $\lambda_{i} \in \boldsymbol{\Lambda}$ where $\boldsymbol{\Lambda}$ is the list of all possible edge labels such that a chromosome is defined by $E=\left\{\lambda_{1}, \lambda_{2}, \cdots \lambda_{N}\right\}$, where $N$ is the number of edges in the drawing. The drawing can be described as a list of junctions $J_{k}, k=1 \cdots K$, where $K$ is the number of junctions. The edge labels $E\left(J_{k}\right) \in E$ therefore list the edge labels 
of edges forming junction $J_{k}$ in chromosome $E$. The fitness of the chromosome is then defined as the Hamming distance between the possible labels defined in junction dictionary $\boldsymbol{\Gamma}$ and $E\left(J_{k}\right)$ in chromosome $E[7]$.

\section{Introducing Drawing Cues to Enhance Line Labeling}

The GA of [7] may converge to a legal labeling which does not match the design intent portrayed by the cues. We enhance this GA such that drawing cues may guide it towards an intended solution. We focus here on three cues, namely cast and attached shadows and table lines which are predominantly used in drawings $[6]$.

Cues constrain the relevant edges to assume a subset of allowed labels. These constraints have been used to compile a cue constraint filter (CCF) to restrict the allowed edge labels of the corresponding edges. This CCF is used to limit the possible edge labels of each gene $g_{n}$ in the chromosomes that form the initial population, thus obtaining an initial population which is close to the expected solution. Since the GA is allowed to change the chromosome through cross-over and mutation operations, the initial information prompted by the cues may be lost through the evolutionary process. For this reason, besides applying the CCF to the initial population, it is also used to obtain a subset of labels $\Lambda(n)$ from $\Lambda$ that may be assigned to a gene $g_{n}$ given the set of cues $C(n)$ that bear upon the edge represented by $g_{n}$. This is represented as $C C F\left(\Lambda \mid g_{n}, C(n)\right)=\Lambda(n)$. We then define a penalty function as $P_{n}=\left\{\frac{1}{N}\right.$ if $\Lambda(n) \neq \varnothing \lambda_{i} \notin \Lambda(n)$; otherwise 0$\}$ which acts as a soft constraint on the edge label. The fitness function is then defined as

$$
F(E)=\alpha\left(\frac{1}{2 N} \sum_{k=1}^{K} \min _{l=1, \cdots,|\boldsymbol{\Gamma}|} H\left(E\left(J_{k}\right), \boldsymbol{\Gamma}\right)\right)-(1-\alpha)\left(\sum_{n=1}^{N} P_{n}\right)
$$

$H$ denotes the Hamming distance and $\alpha$ is a weight factor that determines the confidence in the cues. The value of $\alpha$ was arbitrarily set to 0.6 .

\section{Results and Discussion}

The cue-based GA was evaluated on diagrams such as those shown in Fig. 1 depicting drawings which, although having the same geometric shape, have different cues and require different interpretations. The GA was implemented with proportionate fitness selection, a 1-point crossover with a rate of 0.9 and a mutation rate of 0.03 . The cue-based GA was performed over 50 trials of 500 generations each and performance was compared to a cue-less GA implementation with the same parameters.

In the cue-less GA, all the trials converged to a mean maximum fitness of 1 , achieving geometrically correct solutions in all trials. However, only $18 \%$ achieved the intended solution portrayed by the cues. In contrast, the cue-based GA 


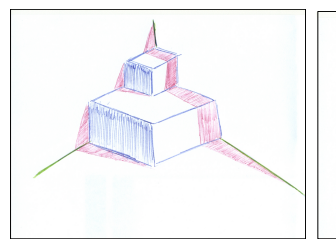

(a)

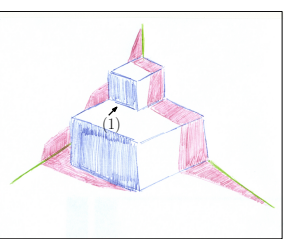

(b)

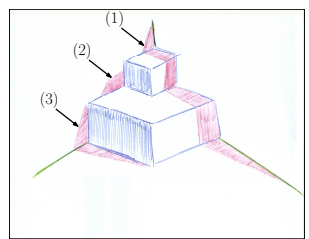

(c)

Fig. 1: A sample of diagrams on which the cue-based GA has been tested. Diagram (b) has a missing cast shadow at (1) and Diagram (c) has conflicting cast shadows at (1), (2) and ( $\underline{\underline{3}}$

achieved a mean maximum fitness of 0.9889 with an average of $76 \%$ converging to the intended solution with the remaining $27 \%$ converging to a solution which, although geometrically correct, was contradictory to the cues. In the case of Fig. 1(c), which is an example of an ambiguous drawing, $46 \%$ of the trials disregarded the evidence of cue (2) which is in conflict with the other cues while the remaining $54 \%$ match all the cues but in so doing create mismatches with the junction dictionary. This experiment shows that the proposed GA may handle ambiguous drawings gracefully.

\section{Conclusion}

The results obtained encourage the use of cues in the interpretation of the drawings and give scope for future work to this effect. One possible improvement to this approach is to make use of a mechanism that would allow the cue-based interpretation of the edge to co-evolve with the junction interpretation of the drawing. This would allow stronger cooperation between the two aspects of the population fitness, hence enhancing the chances of identifying solutions that match the interpretations implied by the cues in the drawing.

\section{References}

[1] Clowes, M.B.: On seeing things. Artificial Intelligence 2(1), 76 - 116 (1971)

[2] Cooper, M.: The interpretation of line drawings with contrast failure and shadows. International Jouranl on Computer Vision 43(2), 75-97 (2001)

[3] Costa Sousa, M., Prusinkiewicz, P.: A few good lines: Suggestive drawing of 3d models. Computer Graphics Forum 22(3), pp. 381-390 (2003)

[4] Guptill, A.L.: Rendering in Pen and Ink. Watson-Guptill (1997)

[5] Huffman, D.A.: Impossible objects as nonsense sentences. Machine Intelligence $6,295-323$ (1971)

[6] Mamassian, P., Knill, D.C., Kersten, D.: The perception of cast shadows. Trends in Cognitive Sciences 2(8), 288-295 (1998)

[7] Myers, R., Hancock, E.R.: Genetic algorithms for ambiguous labelling problems. Pattern Recognition 33(4), 685 - 704 (2000)

[8] Waltz, D.: The Psychology of Computer Vision, chap. 2: Understanding line drawings of scenes with shadows, pp. 19-91. McGraw-Hill (1975) 